\title{
A Rare Case of Nodular Mucinosis of the Breast
}

\author{
Hyun Min Koh · Young Hee Maeng ${ }^{1}$. Bo Geun Jang ${ }^{1} \cdot$ Jae Hyuk Choi ${ }^{2}$. Chang Lim Hyun ${ }^{1}$ \\ Department of Pathology, Jeju National University Hospital, Jeju; Departments of ${ }^{1}$ Pathology and ${ }^{2}$ Surgery, Jeju National University Hospital, \\ Jeju National University College of Medicine and Graduate School of Medicine, Jeju, Korea
}

Nodular mucinosis of the breast is an extremely rare stromal lesion described as a nerve sheath myxoma by Wee et al. ${ }^{1}$ in 1989 , which was the first report of its kind. To the best of our knowledge, the English literature contains few examples of this lesion. In this report, we describe a case of nodular mucinosis characterized by abundant myxoid tissue with scattered spindle cells without epithelial components.

\section{CASE REPORT}

A 22-year-old previously healthy woman visited our hospital complaining of a soft mass on her right breast that had been present for a year. Recently, the lesion had gradually increased in size. She had no other complaints, such as pain or nipple discharge. There was no relevant family or personal medical history. She had never been pregnant and had never taken exogenous hormones or any drugs. The patient showed no evidence of Carney complex. Upon physical examination, a well-demarcated, protruding and movable cystic nodule was present at 10 o'clock on the areola of her right breast. The skin surface covering the nodule exhibited no specific changes and the nipple was unremarkable. Ultrasonography of the breast revealed a 1 -cm ovoid cystic nodule located in the dermal layer, which was presumed to be a benign lesion. Surgical resection was carried out without other diagnostic tests, such as fine needle aspiration or mammography.

Grossly, the specimen was an ovoid cystic nodule measuring $1.1 \times 0.6 \times 0.6 \mathrm{~cm}$ and was covered by an ellipse of normal skin.

\section{Corresponding Author}

Chang lim Hyun, MD, PhD

Department of Pathology, Jeju National University School of Medicine, 15 Aran 13-gil, Jeju 63241, Korea

Tel: +82-64-754-8818, Fax: +82-64-717-1131, E-mail: venisua@jejunu.ac.kr

Received: May 27, 2016 Revised: July 20, 2016

Accepted: July 25, 2016
The outer surface of the nodule was smooth and glistening. Upon sectioning, the cyst was well circumscribed, but not encapsulated (Fig. 1A) and was filled with pale tan, gelatinous material surrounded by grayish white fibrous tissue (Fig. 1B). Histologically, the lesion consisted of abundant myxoid tissue with a few scattered spindle cells and had a slightly multilobular appearance with fibrocollagenous stroma. Capillaries and thin-walled vessels were noted within the nodule (Fig. 1C). Rare histiocytes, lymphocytes, and plasma cells were seen in the nodule. Neither mammary ducts nor epithelial elements were observed. Spindle cells showed bland-looking nuclei and elongated amphophilic cytoplasm with no atypia or mitosis. The myxoid tissue was analyzed via Alcian blue staining ( $\mathrm{pH}$ 2.5) and periodic acid-Schiff (PAS) testing. Alcian blue stained the myxoid stroma bright blue (Fig. 1D) and PAS was negative, indicating the existence of acidic mucopolysaccharides. The spindle cells showed reactivity to vimentin (Fig. 1E) but were nonreactive to smooth muscle actin (Fig. 1F). Based on these findings, we confirmed nodular mucinosis of the breast. Postoperative status was good, and the patient has been in remission for 3 years since the surgery.

\section{DISCUSSION}

Nodular mucinosis of the breast is an extraordinary entity that has been reported fewer than 10 times in the English literature since the first case was described in 1989 (Table 1). ${ }^{1-7}$ Several common clinical and pathologic features were observed in previous reports. Nodular mucinosis of the breast is predominantly found in young women ranging from 15 to 72 years. $^{2-6}$ The typical clinical manifestation is a soft mass under the nipple or in the subareolar region that gradually increases in size, and occasionally presents with nipple discharge or pain. ${ }^{2-6}$ Ultrasonography usually reveals a well-circumscribed, lobulated, homoge- 
nous, hypoechoic mass and mammography shows a roundlobular, radiopaque mass without microcalcifications or spicula formation. ${ }^{2-5}$ Fine needle aspiration typically reveals mucin without epithelial elements or malignant evidence. ${ }^{2,45}$ The treatment of choice is surgical excision, which usually leads to a successful outcome without residual disease. Nodular mucinosis of the breast does not have an established link with Carney complex, which is an autosomal dominant syndrome charac- terized by multiple neoplasias, including myxomas at various sites, endocrine tumors, and lentigenosis related to genetic defects. $^{8}$

Grossly, the lesion is usually a small, ovoid protruding nodule, ranging from 0.9 to $3.2 \mathrm{~cm}$ in diameter. ${ }^{2-6}$ The cut surface demonstrates a well-circumscribed but not encapsulated, multinodular lesion that is grayish-white, grayish-pink, or yellow-to-tan in color $^{2-6}$ and has a myxoid, mucinous or gelatinous consistency. ${ }^{3-6}$
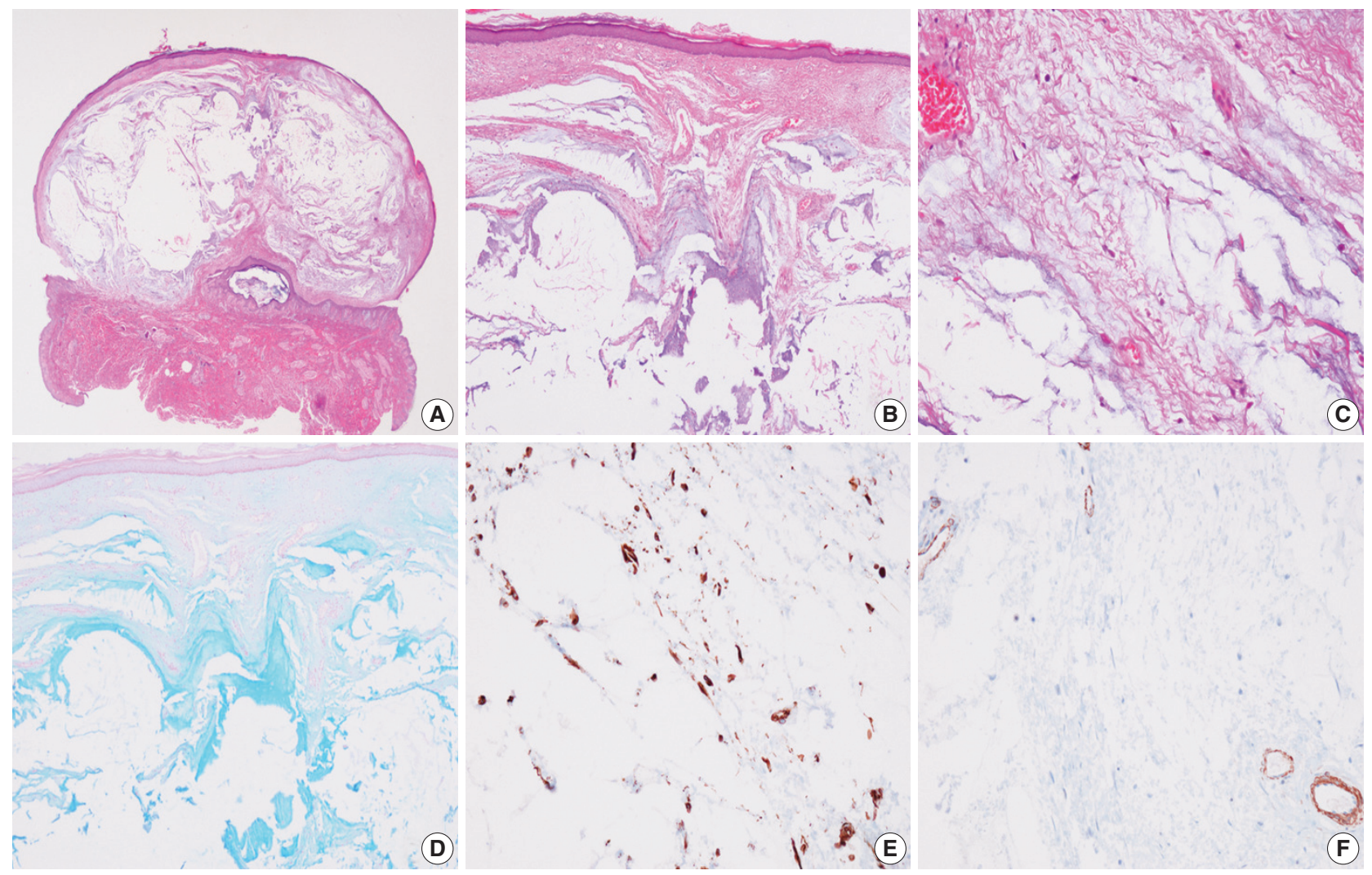

Fig. 1. Histopathologic findings. (A) The lesion is a well-circumscribed cyst showing a slightly multilobular appearance. (B) The lesion is filled with basophilic myxoid substance. (C) The lesion consists of scattered spindle cells with thin walled vessels and capillaries. (D) Alcian blue stains the myxoid material in bright blue. (E) The spindle cells are reactive to vimentin. (F) The spindle cells show nonreactivity to smooth muscle actin.

Table 1. Clinical features of nine reported cases of nodular mucinosis of the breast (including the present case)

\begin{tabular}{|c|c|c|c|c|c|c|}
\hline Reference & Age (yr) & $\mathrm{M} / \mathrm{F}$ & Location & Size $(\mathrm{cm})$ & Follow-up & Prognosis \\
\hline \multirow{3}{*}{ Michal et al..$^{6}$ (1998) } & 40 & M & Left nipple & $2 \times 1.5 \times 1.5$ & $6 \mathrm{yr}$ & No recurrence \\
\hline & 28 & $\mathrm{~F}$ & Right nipple & $1.5 \times 1.5 \times 1$ & $3 \mathrm{yr}$ & No recurrence \\
\hline & 29 & $\mathrm{~F}$ & Right nipple & $2.5 \times 2.5 \times 2$ & $6 \mathrm{mo}$ & No recurrence \\
\hline Koide et al. ${ }^{5}$ (2002) & 30 & $\mathrm{~F}$ & RUO to $N$ & $2.9 \times 2.1$ & NA & NA \\
\hline Sanati et al. ${ }^{4}$ (2005) & 21 & $\mathrm{~F}$ & Left nipple & $1.5 \times 0.9 \times 0.8$ & NA & NA \\
\hline Chisholm et al. ${ }^{3}$ (2010) & 72 & $\mathrm{~F}$ & Right subareolar & $0.9 \times 0.8 \times 0.3$ & $4 \mathrm{mo}$ & No recurrence \\
\hline Manglik et al. ${ }^{2}$ (2010) & 15 & F & $\begin{array}{l}\text { Right subareolar } \\
\text { (supernumerary nipple) }\end{array}$ & $1 \times 1 \times 0.5$ & NA & NA \\
\hline Bulut et al. ${ }^{7}$ (2015) & 37 & $\mathrm{~F}$ & Right subareolar & $3.2 \times 2.5 \times 1.7$ & $29 \mathrm{mo}$ & No recurrence \\
\hline Our case & 22 & $\mathrm{~F}$ & Right subareolar & $1.1 \times 0.6 \times 0.6$ & $3 \mathrm{yr}$ & No recurrence \\
\hline
\end{tabular}

$\mathrm{M}$, male; F, female; RUO to N, right upper outer to nipple; NA, not available. 
The histological features of nodular mucinosis of the breast include a multinodular myxoid or mucinous lesion separated by fibrocollagenous stroma. ${ }^{2-6}$ The lesion consists of sparse spindle cells, which have bland and elongated nuclei, scant and/or pale cytoplasm, and indistinct cell boarders. ${ }^{2,4,5}$ Rarely histiocytes, lymphocytes, plasma cells, and/or mast cells can be seen within and around the nodules, ${ }^{2-6}$ and some capillaries or small vessels are seen within the lesions. ${ }^{3,5,6}$ The lesion neither contains nor invades the breast parenchyma. ${ }^{2-4,6}$ Also, no epithelial components can be identified. ${ }^{2-6}$ Myxoid or mucinous areas are stained intensely with Alcian blue and Hale colloidal iron, but are negative on PAS, proving the existence of acid mucopolysaccharide. ${ }^{2-6}$ The spindle cells are positive for calponin, vimentin, and smooth muscle actin and negative for cytokeratin, smooth muscle myosin, and S-100, probably arising from myofibroblasts. ${ }^{2-5}$

Clinicians need to be able to differentiate nodular mucinosis of the breast from other myxoid and mucinous lesions such as mucinous carcinoma, micropapillary carcinoma in situ with excessive mucin, fibroadenoma with myxomatous stroma and mucocelelike lesions. ${ }^{2-5}$ Nodular mucinosis exhibits mucin with no epithelial elements or malignant evidence on fine needle aspiration., ${ }^{2,4,5}$ The presence of an epithelial cell component in the mucin suggests other myxoid and mucinous lesions. ${ }^{4,7}$ In addition, cytologically mucocele-like lesions are characterized by abundant mucin and monolayer clusters or sheets of epithelial cells without nuclear atypia and is usually associated with microcalcifications, which is a feature not typical of nodular mucinosis. ${ }^{4}$ Mucin in mucocele-like lesions is usually stained with PAS but appears negative on Alcian blue and Hale colloidal iron staining.,

Considering that nodular mucinosis has no epithelial components in acidic mucin pools, differentiation is not a major challenge, so long as spindle cells are not mistaken for epithelial cells.

We have discussed a very rare case of nodular mucinosis of the breast. Although this entity is benign, it is imperative that it be distinguished from other lesions that occur near the nipple, es- pecially mucinous carcinoma, via pathologic examination after surgical excision.

\section{Conflicts of Interest}

No potential conflict of interest relevant to this article was reported.

\section{REFERENCES}

1. Wee A, Tan CE, Raju GC. Nerve sheath myxoma of the breast: a light and electron microscopic, histochemical and immunohistochemical study. Virchows Arch A Pathol Anat Histopathol 1989; 416: 163-7.

2. Manglik N, Berlingeri-Ramos AC, Boroumand N, Eltorky M. Nodular mucinosis of the breast in a supernumerary nipple: case report and review of the literature. J Cutan Pathol 2010; 37: 1178-81.

3. Chisholm C, Greene JF Jr. Nodular mucinosis of the breast: expanding our understanding with an unusual case. Am J Dermatopathol 2010; 32: 187-9.

4. Sanati S, Leonard M, Khamapirad T, Eltorky MA. Nodular mucinosis of the breast: a case report with pathologic, ultrasonographic, and clinical findings and review of the literature. Arch Pathol Lab Med 2005; 129: e58-61.

5. Koide N, Akashi-Tanaka S, Fukutomi T, Nanasawa T, Hasegawa T. Nodular mucinosis of the breast: a case report with clinical and imaging findings. Breast Cancer 2002; 9: 261-4.

6. Michal M, Ludvíková M, Zámecnik M. Nodular mucinosis of the breast: report of three cases. Pathol Int 1998; 48: 542-4.

7. Bulut T, Celik B, Nassar A, Yalcin AD. Nodular mucinosis of breast: a case report. J Med Cases 2015; 6: 457-9.

8. Stratakis CA, Carney JA, Lin JP, et al. Carney complex, a familial multiple neoplasia and lentiginosis syndrome: analysis of 11 kindreds and linkage to the short arm of chromosome 2. J Clin Invest 1996; 97: 699-705. 